\title{
Insect galls of Restinga de Marambaia (Barra de Guaratiba, Rio de Janeiro, RJ)
}

\author{
V. C. Maia ${ }^{a *}$ and L. O. Silva ${ }^{a}$ \\ ${ }^{a}$ Museu Nacional, Quinta da Boa Vista, São Cristóvão, CEP 20940-040, Rio de Janeiro, RJ, Brazil \\ *e-mail: maiavcid@acd.ufrj.br
}

Received: April 16, 2015 - Accepted: May 21, 2015 - Distributed: August 31, 2016

(With 37 figures)

\begin{abstract}
Thirty-one morphotypes of insect galls and two flower damages were found on 16 families, 22 genera and 24 plant species in Restinga de Marambaia (Barra de Guaratiba, Rio de Janeiro, RJ). Fabaceae and Myrtaceae were the plant families with the greatest richness of insect galls (4 and 6 morphotypes, respectively), and the greatest number of galled plants (four and three species, respectively). Galls were mostly found on leaves and stems (77\% and 10\%, respectively). The galling insects are represented by Diptera, Lepidoptera, and Hemiptera. The majority of the galls (81\%) were induced by gall midges (Cecidomyiidae: Diptera).
\end{abstract}

Keywords: Cecidomyiidae, Diptera, insect-plant interaction, inventory.

\section{Galhas de insetos da Restinga de Marambaia (Barra de Guaratiba, Rio de Janeiro, RJ)}

\section{Resumo}

Foram encontrados 31 morfotipos de galhas de insetos e dois danos em flores em 16 famílias, 22 gêneros e 24 espécies de plantas, na Restinga de Marambaia (Barra de Guaratiba, Rio de Janeiro, RJ). Fabaceae e Myrtaceae foram as famílias de plantas com maior riqueza de galhas (4 e 6 morfotipos, respectivamente) e maior número de espécies com galhas (quatro e três espécies, respectivamente). As galhas predominaram nas folhas e caules ( $77 \%$ e $10 \%$, respectivamente). Os insetos galhadores estão representados por Diptera, Lepidoptera e Hemiptera. A maioria das galhas (81\%) foi induzida por Cecidomyiidae (Diptera).

Palavras-chave: Cecidomyiidae, Diptera, interação inseto-planta, inventário.

\section{Introduction}

Insect galls are characterized by abnormal growth of plant tissues and involve cell hypertrophy and/or hyperplasia (Mani, 1964). The galling insects have the ability to manipulate the development of plant tissue and promote its growth (Stone and Schönrogge, 2003) and the development of these structures has been an adaptive strategy of many insects for their food and even protection against predators (Mani, 1964; Stone and Schönrogge, 2003). Therefore, galls are considered the most sophisticated insect-plant interaction (Shorthouse et al., 2005).

In the State of Rio de Janeiro, several insect galls inventories have been published in restinga areas, in the following localities: Arraial do Cabo (APA de Massambaba, Monteiro et al., 1994 and Ilha do Cabo Frio, Maia and Souza, 2013), Jurubatiba (Monteiro et al., 2004), Carapebus and Maricá (Maia, 2001), Grumari (Rio de Janeiro) (Oliveira and Maia, 2005), and Reserva Biológica Estadual da Praia do Sul (Ilha Grande, Angra dos Reis) (Maia and Oliveira, 2010). Furthermore, some records of

insect galls are known from Parque Municipal da Boca da Barra (Cabo Frio, Rodrigues and Silva, 2011), and Paraty (Fernandes and Maia, 2011). These inventories indicate a great richness of insect galls in this ecosystem. Data on Restinga de Marambaia is still unknown. The objective of this work is to survey and characterize the insect galls of Restinga de Marambaia.

\section{Material and Methods}

The Restinga de Marambaia (Figure 1) has a total area of $81 \mathrm{Km}^{2}$ and spreads over three municipalities of Rio de Janeiro State: Rio de Janeiro, Itaguaí, and Mangaratiba (CGCFN, 2015). It is separated from the continent by the Canal do Bacalhau (Figure 1). It is considered an area of special environmental interest (Rio de Janeiro, 2013), being under the control of the Brazilian Navy and Army. It is included in Atlantic Forest biome, and comprises three ecosystems: mangrove, restinga, and ombrophilous forest 


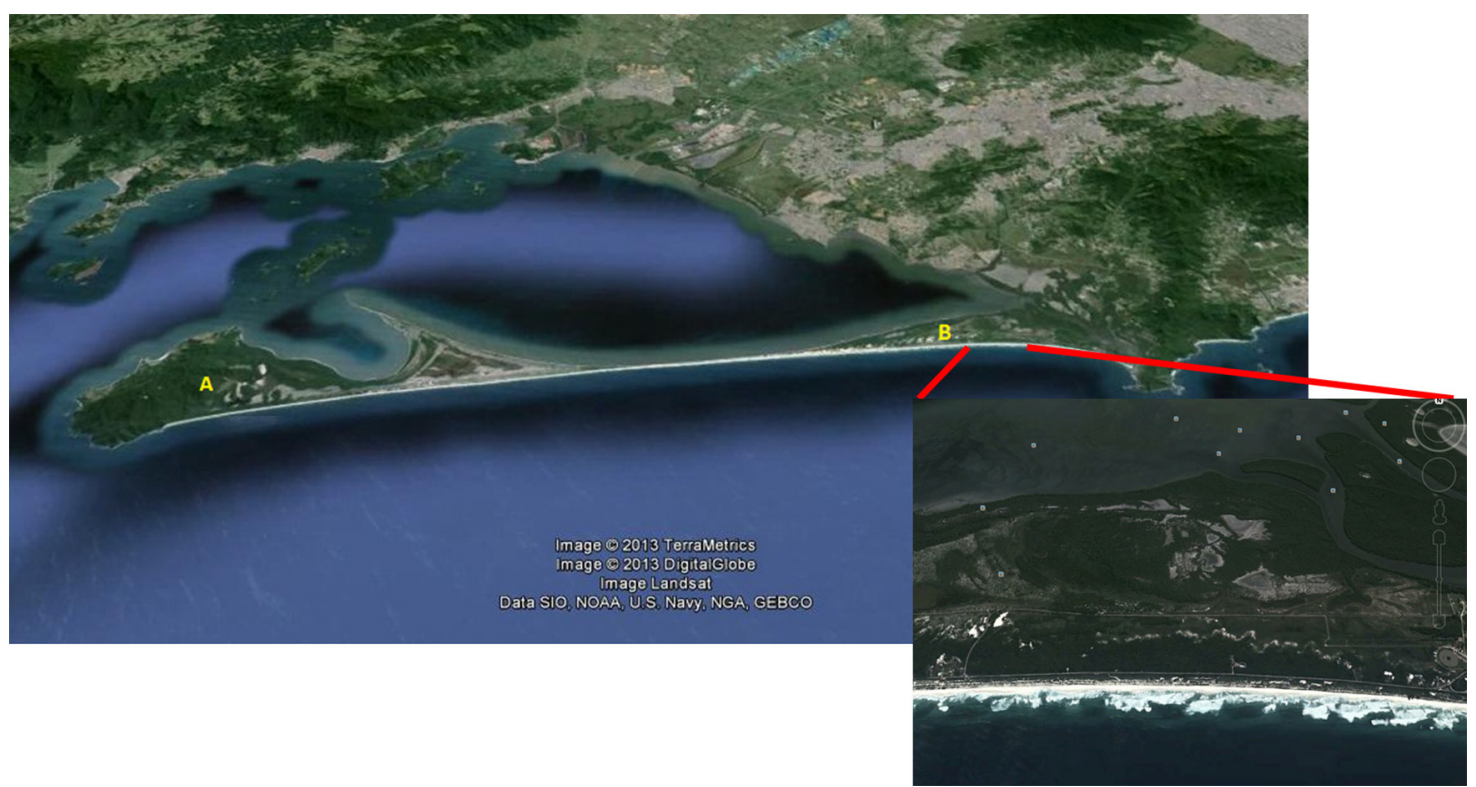

Figure 1. Ilha de Marambaia, Mangaratiba (A) e Restinga de Marambaia, Barra de Guaratiba (B). Increased passage about Line 1. Google Earth, 22.X.2013.

(Afonso et al., 2007). According to Köppen classification, the macroclimate is AW (rainy tropical).

The studied area is situated in the eastern portion of the Restinga da Marambaia, in the municipality of Rio de Janeiro, near Barra de Guaratiba. Twenty four sites were established along the pathway known as "line 1 " $\left(23^{\circ} 02\right.$ ' $\left.56^{\prime \prime} \mathrm{S}, 43^{\circ} 37^{\prime} 51^{\prime \prime} \mathrm{W}\right)$ at every 300 meters, totalizing $6.2 \mathrm{Km}$ of surveyed area. Each site investigated for 30 minutes by two people, monthly, from January to May of 2012, totalizing 120 hours.

According to Afonso et al. (2007) the open shrubby formation of line 1 comprises 48 plant species in 43 genera and 24 families. They indicated the Fabaceae, Myrtaceae, Polygonaceae, Sapindaceae, and Sapotaceae as the richest families in number of species, and Coccoloba Vell. and Eugenia Casar. as the most speciose genera.

All plant organs were examined, except for the subterraneous roots. The plants were identified by the first author. All gall morphotypes were photographed, using a digital camera. Each morphotype was characterized based on shape (according to Isaias et al., 2013), plant organ, color, presence or absence of trichomes, and number of internal chambers. For each gall morphotype, previous records from other restinga inventories are provided.

To obtain the galling insects, each gall morphotype was individually kept in labeled plastic pots layered at the bottom with damp cotton and covered by fine screening. All pots were checked daily for emergence. The specimens were preserved in 70\% alcohol (Gagné, 1994). The gall midges were identified by the authors based on the gall morphology, host plant and original descriptions. All material is deposited in the entomological collection of Museu Nacional/Universidade Federal do Rio de Janeiro (MNRJ).

\section{Results}

A total of 31 insect gall morphotypes and two flower damages were found in 16 families, 22 genera and 24 plant species in this inventory (Figures 2-37). The medium number of gall morphotypes and damages per plant species was 1.3. Three gall morphotypes were collected in magroves and 28 in the sandbank. Eugenia uniflora L. was observed in both ecosystems, hosting different gall morphotypes (conical on mangroves and circular on sandbanks) and, therefore, induced by different species of insects.

Fabaceae and Myrtaceae were the plant families with the greatest richness of gall (4 and 6 morphotypes, respectively), and the greatest number of galled plant species (four and three, respectively) (Table 1).

The gall morphotypes characterization is presented in Table 2. About $77 \%$ of the galls occurred on leaves. Stem (10\%), flower bud (7\%), bud (3\%) and fruit (3\%) galls were also found. All gall morphotypes showed plant organ specifity, excepting the conical galls on Erythroxylum ovalifolium Peyr. (Erythroxylaceae), and the globoid galls on Byrsonima sericea DC. (Malpighiaceae), both occurring on leaves and stems (Table 3).

Besides the galling species, two free-living larvae were found, one on flowers of Asteraceae (not determined species), and the other, Clinodiplosis floricola Novo-Guedes and Maia, 2008 on bud flowers of Heteropteris nitida Juss (Malpighiaceae).

We observed twelve different gall shapes (Table 4). Among these, the most frequent were discoid (23\%), globoid (20\%), and conical (16\%).

Most galls are glabrous (97\%), 87\% were one-chambered, and $87 \%$ presented the same color of the galled organ, with four exceptions: the leaf galls of Clinodiplosis sp. 


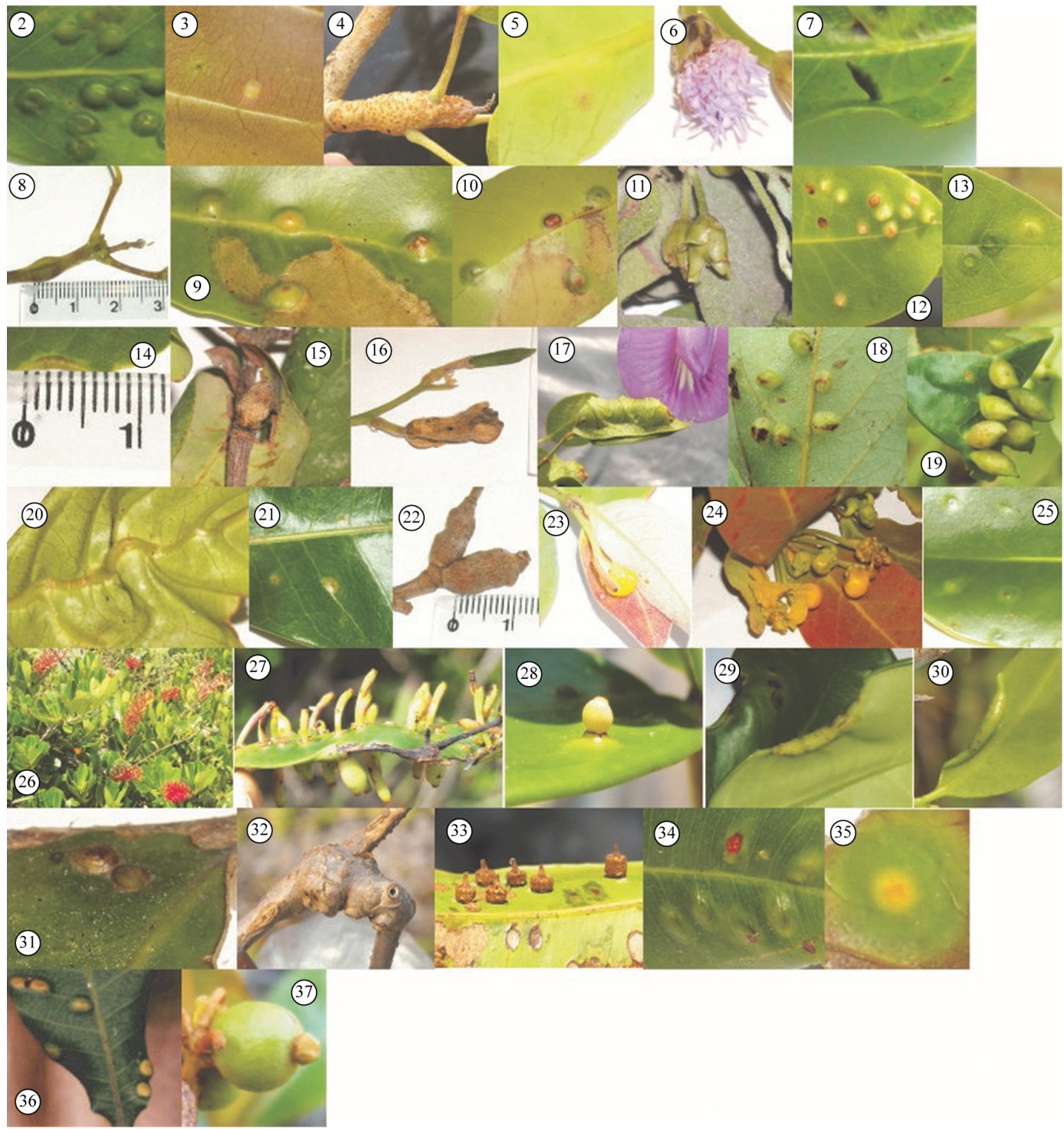

Figures 2-37. Insect galls from Restinga de Marambaia (Barra de Guaratiba, Rio de Janeiro, RJ). (2) Intralaminar globoid leaf gall on Avicennia sp., galler Cecidomyiidae; (3) Discoid leaf gall on Anacardium occidentale, galler Cecidomyiidae; (4) Fusiform stem gall on Schinus terebinthifolius, galler Lepidoptera; (5) Discoid leaf gall on Aspidosperma pyricollum, galler Psyllidae (Hemiptera); (6) Damage on flower of Asteraceae (not determined), insect not determined(Free larva); (7)Conical leaf gall on Fridericia conjugata, galler Arrabidaeamyia serrata; (8) Globoid stem gall on F. conjugata, galler Neolasioptera sp.; (9) Intralaminar lenticular leaf gall on Maytenus obtusifolia in adaxial surface, galler Mayteniella distincta; (10) The same, abaxial surface; (11) Globoid discrete bud flower gall on Jacquemontia holosericea, galler Schizomyia santosi; (12) Conical leaf gall on Erythroxylum ovalifolium, galler Dasineura ovalifoliae; (13) Circular leaf gall on Eugenia uniflora, galler Neolasioptera engeniae; (14) Marginal leaf roll gall on E. ovalifolium, galler Clinodiplosis sp.; (15) Conical bud gall on E. ovalifolium, galler Lopesia erythroxyli; (16) Conical bud flower gall on Clitoria laurifolia, galler not determined; (17) Globoid leaf gall on Clitoria ternatea, galler not determined; (18) Discoid leaf gall on Dalbergia ecastophylla, galler Lopesia grandis; (19) Conical leaf gall on E. uniflora, galler Clinodiplosis profusa; (20) Pocket shaped leaf gall on Inga maritima, galler Neolasioptera ingae; (21) Discoid leaf gall on Byrsonima sericea, galler Dasineura byrsonimae; (22) Globoid stem gall on B. sericea, galler Bruggmanniella byrsonimae; (23 and 24) Bud flower of Heteropterys nitida, free larva of the Clinodiplosis floricola; (25) Intralaminar lenticular leaf gall on Norantea brasiliensis, galler not determined; (26) N. brasiliensis in loco; (27) Clavate leaf gall on Eugenia astringens, galler Stephomyia clavata; (28) Globoid leaf gall on E. astringens, galler Dasineura globosa; (29) Marginal leaf roll gall on E. astringens, galler Dasineura marginalis; (30) Marginal leaf roll gall on Neomitranthes obscura, galler Clinodiplosis sp.; (31) Intralaminar lenticular leaf gall on Guapira opposita, galler Bruggmannia elongata; (32) Globoid stem gall on G. opposita, galler Proasphondylia guapirae; (33) Cylindrical with apical projection leaf gall on Ouratea cuspidata, galler Contarinia sp.; (34) Discoid leaf gall on Manilkara subsericea, galler Manilkaramyia notabilis; (35) Discoid leaf gall on Smilax rufescens, galler Smilasioptera candelariae; (36) Discoid leaf gall on Pouteria venosa, galler Lopesia singularis; (37) Globoid leaf gall on Byrsonima sericea, galler Lepidoptera. 
Table 1. Distribution of the number of morphotypes of insect galls and damages per host plant and ecosystem in the Restinga de Marambaia (Barra de Guaratiba, Rio de Janeiro, RJ).

\begin{tabular}{|c|c|c|c|}
\hline Plant family & Plant species & $\begin{array}{c}\text { Number of galls } \\
\text { and damage }\end{array}$ & Ecosystem \\
\hline Acanthaceae & $\begin{array}{l}\text { Avicennia schaueriana Stapf and Leechm. } \\
\text { ex Moldenke }\end{array}$ & 1 & Mangrove \\
\hline \multirow[t]{2}{*}{ Anacardiaceae } & Anacardium occidentale L. & 1 & Restinga \\
\hline & Schinus terebinthifolius Raddi. & 1 & Restinga \\
\hline Apocynaceae & Aspidosperma pyricollum M. Arg. & 1 & Restinga \\
\hline Asteraceae & Not determined & 1 & Mangrove \\
\hline Bignoniaceae & Fridericia conjugata (Vell.) Mart. & 2 & Restinga \\
\hline Celastraceae & Maytenus obtusifolia Mart. & 1 & Restinga \\
\hline Convolvulaceae & Jacquemontia holosericea (Weinm.) O'Donell. & 1 & Restinga \\
\hline Erythroxylaceae & Erythroxylum ovalifolium Peyr. & 3 & Restinga \\
\hline \multirow[t]{4}{*}{ Fabaceae } & Clitoria laurifolia Poir. & 1 & Restinga \\
\hline & Clitoria ternatea $\mathrm{L}$. & 1 & Restinga \\
\hline & Dalbergia ecastophylla L. Taub & 1 & Mangrove \\
\hline & Inga maritima Benth. & 1 & Restinga \\
\hline \multirow[t]{2}{*}{ Malpighiaceae } & Byrsonima sericea DC. & 3 & Restinga \\
\hline & Heteropterys nitida Juss. & 1 & Restinga \\
\hline Marcgraviaceae & Norantea brasiliensis Choisy. & 1 & Restinga \\
\hline \multirow[t]{3}{*}{ Myrtaceae } & Eugenia astringens Cambess. & 3 & Restinga \\
\hline & Eugenia uniflora L. & 2 & $\begin{array}{l}\text { Mangrove and } \\
\text { Restinga }\end{array}$ \\
\hline & Neomitranthes obscura (DC) N. Silveira & 1 & Restinga \\
\hline Nyctaginaceae & Guapira opposita (Vell.) Reitz. & 2 & Restinga \\
\hline Ochnaceae & Ouratea cuspidata (St. Hil) Engl. & 1 & Restinga \\
\hline \multirow[t]{2}{*}{ Sapotaceae } & Manilkara subsericea (Mart.) Dubard. & 1 & Restinga \\
\hline & Pouteria venosa (Mart.) Baehni. & 1 & Restinga \\
\hline Smilacaceae & Smilax rufescens Griseb & 1 & Restinga \\
\hline
\end{tabular}

Table 2. Characterization of insect galls of the Restinga of Marambaia (Barra de Guaratiba, Rio de Janeiro, RJ).

\begin{tabular}{|c|c|c|c|c|c|c|}
\hline Plant species & Gall shape & $\begin{array}{l}\text { Gall color / } \\
\text { Plant organ }\end{array}$ & Trichomes & $\begin{array}{l}\text { Number } \\
\text { internal } \\
\text { chamber }\end{array}$ & Galler & $\begin{array}{l}\text { Previous } \\
\text { records }\end{array}$ \\
\hline A. schaueriana & $\begin{array}{l}\text { Intralaminar } \\
\text { globoid }\end{array}$ & Green / Leaf & Absent & 1 & Cecidomyiidae & $\begin{array}{l}\mathrm{BER} \text { and } \\
\mathrm{CF}\end{array}$ \\
\hline A. occidentale & Discoid & Green / Leaf & Absent & 1 & Cecidomyiidae & AMA \\
\hline S. terebinthifolius & Fusiform & $\begin{array}{c}\text { Brown / } \\
\text { Stem }\end{array}$ & Absent & 1 & Lepidoptera & $\begin{array}{l}\text { BER, GRU } \\
\text { and } \mathrm{CF}\end{array}$ \\
\hline A. pyricollum & Discoid & Green / Leaf & Absent & 1 & $\begin{array}{l}\text { Psyllidae } \\
\text { (Hemiptera) }\end{array}$ & $\begin{array}{l}\text { IG and } \\
\text { MAR }\end{array}$ \\
\hline Asteraceae & Free larva & - / Fower & - & - & Not determined & - \\
\hline \multirow[t]{2}{*}{ F. conjugata } & Conical & Green / Leaf & Absent & 1 & $\begin{array}{l}\text { Arrabidaeamyia } \\
\text { serrata } \text { Maia, } 2001 \\
\text { Cecidomyiidae }\end{array}$ & $\begin{array}{l}\text { GRU, } \\
\text { MAR, AC, } \\
\text { CAR and } \\
\text { JU }\end{array}$ \\
\hline & Globoid & Green / Fruit & Absent & Several & $\begin{array}{l}\text { Neolasioptera sp. } \\
\text { Cecidomyiidae }\end{array}$ & $\begin{array}{l}\text { GRU, AR, } \\
\text { CAR and } \\
\text { JU }\end{array}$ \\
\hline
\end{tabular}


Table 2. Continued...

\begin{tabular}{|c|c|c|c|c|c|c|}
\hline Plant species & Gall shape & $\begin{array}{l}\text { Gall color / } \\
\text { Plant organ }\end{array}$ & Trichomes & $\begin{array}{c}\text { Number } \\
\text { internal } \\
\text { chamber }\end{array}$ & Galler & $\begin{array}{l}\text { Previous } \\
\text { records }\end{array}$ \\
\hline M. obtusifolia & $\begin{array}{l}\text { Intralaminar } \\
\text { lenticular }\end{array}$ & Green / Leaf & Absent & 1 & $\begin{array}{l}\text { Mayteniella } \\
\text { distincta } \text { Maia, } 2001 \\
\text { Cecidomyiidae }\end{array}$ & $\begin{array}{l}\text { GRU, } \\
\text { MAR and } \\
\text { AC }\end{array}$ \\
\hline J. holosericea & $\begin{array}{l}\text { Globoid } \\
\text { discrete }\end{array}$ & $\begin{array}{l}\text { Green / Bud } \\
\text { flower }\end{array}$ & Absent & 1 & $\begin{array}{l}\text { Schizomyia santosi } \\
\text { Maia and Araújo, } \\
2009 \text { Cecidomyiidae }\end{array}$ & MAR \\
\hline \multirow[t]{3}{*}{ E. ovalifolium } & Conical & Green / Leaf & Absent & 1 & $\begin{array}{l}\text { Dasineura } \\
\text { ovalifoliae Maia } \\
\text { and Fernandes, } 2011 \\
\text { Cecidomyiidae }\end{array}$ & $\begin{array}{l}\text { GRU } \\
\text { MAR, CAR } \\
\text { and JU }\end{array}$ \\
\hline & $\begin{array}{l}\text { Marginal } \\
\text { Leaf roll }\end{array}$ & $\begin{array}{l}\text { Light green / } \\
\text { Leaf }\end{array}$ & Absent & 1 & $\begin{array}{l}\text { Clinodiplosis sp. } \\
\text { Cecidomyiidae }\end{array}$ & $\begin{array}{l}\text { IG, MAR, } \\
\text { CAR and } \\
\text { JU }\end{array}$ \\
\hline & Conical & Brown / Bud & Absent & 1 & $\begin{array}{l}\text { Lopesia erythroxyli } \\
\text { Rodrigues and Maia } \\
(2010)\end{array}$ & $\begin{array}{l}\text { IG, GRU, } \\
\text { MAR, AC, } \\
\text { CAR and } \\
\text { JU }\end{array}$ \\
\hline C. laurifolia & Conical & $\begin{array}{l}\text { Brown / Bud } \\
\text { flower }\end{array}$ & Present & 1 & Not determined & - \\
\hline C. ternatea & Globoid & Green / Leaf & Absent & 1 & Not determined & - \\
\hline D. ecastophylla & Discoid & Green / Leaf & Absent & 1 & $\begin{array}{l}\text { Lopesia grandis } \\
\text { Maia, 2001a } \\
\text { Cecidomyiidae }\end{array}$ & $\begin{array}{l}\text { IG, MAR, } \\
\text { CAR and } \\
\text { JU }\end{array}$ \\
\hline I. maritima & $\begin{array}{l}\text { Pocket } \\
\text { shaped }\end{array}$ & Green / Leaf & Absent & 1 & $\begin{array}{l}\text { Neolasioptera } \\
\text { ingae Möhn, 1964a } \\
\text { Cecidomyiidae }\end{array}$ & $\begin{array}{l}\text { GRU and } \\
\text { MAR }\end{array}$ \\
\hline \multirow[t]{3}{*}{ B. sericea } & Discoid & $\begin{array}{c}\text { Green or } \\
\text { yellow / Leaf }\end{array}$ & Absent & 1 & $\begin{array}{l}\text { Dasineura } \\
\text { byrsonimae Maia, } \\
2010 \text { Cecidomyiidae }\end{array}$ & $\begin{array}{l}\text { IG, GRU } \\
\text { MAR, } \\
\text { CAR, JU } \\
\text { and GUA }\end{array}$ \\
\hline & Globoid & $\begin{array}{l}\text { Brown / } \\
\text { Stem }\end{array}$ & Absent & Several & $\begin{array}{l}\text { Bruggmanniella } \\
\text { byrsonimae Maia } \\
\text { and Couri, } 1992 \\
\text { Cecidomyiidae } \\
\end{array}$ & $\begin{array}{l}\text { MAR, CAR } \\
\text { and JU }\end{array}$ \\
\hline & Globoid & Green / Leaf & Absent & 1 & Lepidoptera & $\begin{array}{l}\text { MAR and } \\
\text { AC }\end{array}$ \\
\hline H. nitida & Free larva & $\begin{array}{l}\text { - / Bud } \\
\text { flower }\end{array}$ & - & - & $\begin{array}{l}\text { Clinodiplosis } \\
\text { floricola Novo- } \\
\text { Guedes and Maia, } \\
2008 \text { Cecidomyiidae }\end{array}$ & MAR \\
\hline N. brasiliensis & $\begin{array}{c}\text { Intralaminar } \\
\text { lenticular }\end{array}$ & Green / Leaf & Absent & 1 & Not determined & - \\
\hline \multirow[t]{2}{*}{ E. astringens } & Clavate & Green / Leaf & Absent & 1 & $\begin{array}{l}\text { Stephomyia clavata } \\
\text { Tavares, } 1920 \\
\text { Cecidomyiidae }\end{array}$ & $\begin{array}{l}\text { CAR and } \\
\text { JU }\end{array}$ \\
\hline & Globoid & $\begin{array}{l}\text { Yellow / } \\
\text { Leaf }\end{array}$ & Absent & 1 & $\begin{array}{l}\text { Dasineura globosa } \\
\text { Maia, } 1995 \\
\text { Cecidomyiidae }\end{array}$ & $\begin{array}{l}\text { GRU, } \\
\text { MAR, CAR } \\
\text { and JU }\end{array}$ \\
\hline
\end{tabular}

AC = Arraial do Cabo (RJ) (Monteiro et al., 1994); BER = Bertioga (SP) (Maia et al., 2008); CAR = Carapebus (RJ) (Maia, 2001); $\mathrm{CF}=$ Cabo Frio (RJ) (Rodrigues and Silva, 2011; Maia and Souza, 2013); GRU = Grumari (RJ) (Oliveira and Maia, 2005); GUA = Guarapari (ES) (Bregonci et al., 2010); IG = Ilha Grande (RJ) (Maia and Oliveira, 2010); JU = Jurubatiba (RJ); MAR = Maricá (RJ) (Maia, 2001); PA = Paraty (RJ) (Fernandes and Maia, 2011). 
Table 2. Continued...

\begin{tabular}{|c|c|c|c|c|c|c|}
\hline Plant species & Gall shape & $\begin{array}{l}\text { Gall color / } \\
\text { Plant organ }\end{array}$ & Trichomes & $\begin{array}{l}\text { Number } \\
\text { internal } \\
\text { chamber }\end{array}$ & Galler & $\begin{array}{l}\text { Previous } \\
\text { records }\end{array}$ \\
\hline & $\begin{array}{l}\text { Marginal } \\
\text { Leaf roll }\end{array}$ & Green / Leaf & Absent & 1 & $\begin{array}{l}\text { Dasineura } \\
\text { marginalis } \\
\text { Maia, 2005a } \\
\text { Cecidomyiidae }\end{array}$ & $\begin{array}{l}\text { GRU, } \\
\text { MAR, AC, } \\
\text { CAR and } \\
\text { JU }\end{array}$ \\
\hline \multirow[t]{2}{*}{ E. uniflora } & Conical & Green / Leaf & Absent & 1 & $\begin{array}{l}\text { Clinodiplosis } \\
\text { profusa Maia, 2001a } \\
\text { Cecidomyiidae }\end{array}$ & $\begin{array}{l}\text { GRU, } \\
\text { MAR, AC, } \\
\text { CF, CAR } \\
\text { and JU }\end{array}$ \\
\hline & Circular & Green / Leaf & Absent & 1 & $\begin{array}{l}\text { Neolasioptera } \\
\text { eugeniae Maia, } 1993 \\
\text { Cecidomyiidae }\end{array}$ & $\begin{array}{l}\text { PA, IG, } \\
\text { GRU, } \\
\text { MAR, CF } \\
\text { and AC }\end{array}$ \\
\hline N. obscura & $\begin{array}{l}\text { Marginal } \\
\text { Leaf roll }\end{array}$ & Green / Leaf & Absent & 1 & $\begin{array}{l}\text { Clinodiplosis sp. } \\
\text { Cecidomyiidae }\end{array}$ & $\begin{array}{l}\text { MAR, } \\
\text { CAR, JU } \\
\text { and GUA }\end{array}$ \\
\hline \multirow[t]{2}{*}{ G. opposita } & $\begin{array}{c}\text { Intralaminar } \\
\text { lenticular }\end{array}$ & Green / Leaf & Absent & 1 & $\begin{array}{l}\text { Bruggmannia } \\
\text { elongata Maia } \\
\text { and Couri, } 1993 \\
\text { Cecidomyiidae }\end{array}$ & $\begin{array}{l}\text { BER, IG, } \\
\text { MAR, AC, } \\
\text { CAR and } \\
\text { JU }\end{array}$ \\
\hline & Globoid & $\begin{array}{l}\text { Brown / } \\
\text { Stem }\end{array}$ & Absent & Several & $\begin{array}{l}\text { Proasphondylia } \\
\text { guapirae } \\
\text { Maia, 1994a } \\
\text { Cecidomyiidae }\end{array}$ & $\begin{array}{l}\text { BER, IG, } \\
\text { MAR, CAR } \\
\text { and JU }\end{array}$ \\
\hline O. cuspidata & $\begin{array}{l}\text { Cylindrical } \\
\text { with apical } \\
\text { projection }\end{array}$ & Brown / Leaf & Absent & 1 & $\begin{array}{l}\text { Contarinia sp. } \\
\text { Cecidomyiidae }\end{array}$ & $\begin{array}{l}\text { IG, GRU, } \\
\text { MAR, JU } \\
\text { and GUA }\end{array}$ \\
\hline M. subsericea & Discoid & Green / Leaf & Absent & $1-2$ & $\begin{array}{l}\text { Manilkaramyia } \\
\text { notabilis Maia, } 2001 \\
\text { Cecidomyiidae }\end{array}$ & $\begin{array}{l}\text { CAR, JU } \\
\text { and GUA }\end{array}$ \\
\hline P. venosa & Discoid & Green / Leaf & Absent & 1 & $\begin{array}{l}\text { Lopesia singularis } \\
\text { Maia, } 2001 \\
\text { Cecidomyiidae }\end{array}$ & $\begin{array}{l}\text { BER, MAR } \\
\text { and AC }\end{array}$ \\
\hline S. rufescens & Discoid & Green / Leaf & Absent & 1 & $\begin{array}{l}\text { Smilasioptera } \\
\text { candelariae Möhn, } \\
1975 \text { Cecidomyiidae }\end{array}$ & $\begin{array}{l}\text { GRU, } \\
\text { MAR, CAR } \\
\text { and JU }\end{array}$ \\
\hline
\end{tabular}

$\mathrm{AC}=$ Arraial do Cabo (RJ) (Monteiro et al., 1994); BER = Bertioga (SP) (Maia et al., 2008); CAR = Carapebus (RJ) (Maia, 2001); CF = Cabo Frio (RJ) (Rodrigues and Silva, 2011; Maia and Souza, 2013); GRU = Grumari (RJ) (Oliveira and Maia, 2005); GUA = Guarapari (ES) (Bregonci et al., 2010); IG = Ilha Grande (RJ) (Maia and Oliveira, 2010); JU = Jurubatiba (RJ); MAR = Maricá (RJ) (Maia, 2001); PA = Paraty (RJ) (Fernandes and Maia, 2011).

Table 3. Number of insect galls by galled plant organ in the Restinga of Marambaia (Barra de Guaratiba, Rio de Janeiro, RJ).

\begin{tabular}{cc}
\hline Plant organ & $\begin{array}{c}\text { Number of gall morphotypes } \\
(\mathbf{N}=\mathbf{3 1})\end{array}$ \\
\hline Leaf & $24(\approx 77 \%)$ \\
Stem & $3(\approx 10 \%)$ \\
Bud flower & $2(\approx 7 \%)$ \\
Bud & $1(\approx 3 \%)$ \\
Fruit & $1(\approx 3 \%)$ \\
\hline
\end{tabular}

on Erythroxylum ovalifolium Peyr. (Erythroxylaceae), the bud flower galls on Clitoria laurifolia Poir. (Fabaceae), the leaf galls of Dasineura globosa Maia, 1995 on Eugenia astringens Cambess. (Myrtaceae), and the leaf galls of Contarinia sp. on Ouratea cuspidata (St. Hil) Engl. (Ochnaceae). The galled organs were green, but the galls were light green, brown, yellow, and brown, respectively.

The galls were induced by three insect orders: Diptera (Cecidomyiidae), Hemiptera, and Lepidoptera. Cecidomyiidae (Diptera) were the most frequent gallers, inducing $81 \%$ of the morphotypes (Table 5). 
Table 4. Number of insect galls by gall shape in the Restinga of Marambaia (Barra de Guaratiba, Rio de Janeiro, RJ).

\begin{tabular}{lc}
\hline \multicolumn{1}{c}{ Gall shape } & $\begin{array}{c}\text { Number of gall } \\
\text { morphotypes }(\mathbf{N}=\mathbf{3 1})\end{array}$ \\
\hline Discoid & $7(\approx 23 \%)$ \\
Globoid & $6(\approx 20 \%)$ \\
Conical & $5(\approx 16 \%)$ \\
Marginal leaf roll & $3(\approx 10 \%)$ \\
Intralaminar lenticular & $3(\approx 10 \%)$ \\
Fusiform & $1(\approx 3 \%)$ \\
Intralaminar globoid & $1(\approx 3 \%)$ \\
Circular & $1(\approx 3 \%)$ \\
Clavate & $1(\approx 3 \%)$ \\
Pocket shaped & $1(\approx 3 \%)$ \\
Globoid discrete & $1(\approx 3 \%)$ \\
Cylindrical with apical & $1(\approx 3 \%)$ \\
projection & \\
\hline
\end{tabular}

Table 5. Number of insect galls by galling insect in the Restinga of Marambaia (Barra de Guaratiba, Rio de Janeiro, $\mathrm{RJ})$.

\begin{tabular}{lc}
\hline \multicolumn{1}{c}{ Galling insect } & $\begin{array}{c}\text { Number of gall } \\
\text { morphotypes }(\mathbf{N}=\mathbf{3 1})\end{array}$ \\
\hline Diptera (Cecidomyiidae) & $25(\approx 81 \%)$ \\
Not determined & $3(\approx 10 \%)$ \\
Lepidoptera & $2(\approx 6 \%)$ \\
Hemiptera & $1(\approx 3 \%)$ \\
\hline
\end{tabular}

\section{Discussion}

Thirty-one insect gall morphotypes and two flower damages were found in the studied area. Previous inventories in restinga areas of the State of Rio de Janeiro have reported values ranging from 101 (Maia, 2001) to 36 (Maia and Oliveira, 2010), but the sampling methods were not standardized: some restingas were surveyed for 12 months, whereas others for three. In spite of the restinga of Marambaia was surveyed for five months, new gall morphotypes were not found in the last three months, so we believe that the area was sufficiently investigated.

The great difference in the number of gall morphotypes between the two studied ecosystems (mangroove and sandbank) can be explained by the great difference in the diversity of plant species, being sandbanks much richer than mangrooves.

According to previous studies, the medium number of gall morphotypes in restinga areas of the State of Rio varies from 2.1 (Maia, 2001) to 1.5 (Maia and Souza, 2013). The value of the restinga de Marambaia is the lowest one (1.3), what can be related to the low number of botanical species along the investigated area (line 1): 48 species (Afonso et al., 2007).

The majority of the galls were induced by Cecidomyiidae (81\%). As the Cecidomyiidae cause about $70 \%$ of all described galls in the world, these results were expected. Although galls of Coleoptera, Thysanoptera, and Hymenoptera have been recorded in other Brazilian restinga areas (Maia, 2013), they were not found in the investigated area of the Restinga of Marambaia.

There was a predominance of galls on Fabaceae and Myrtaceae (four and six morphotypes, respectively). Fabaceae and Myrtaceae showed a higher number of species plants with gall (four and three, respectively). Maia (2013) indicated the predominance of galls on Myrtaceae, Asteraceae and Fabaceae for restinga vegetation of the Southeastern region of Brazil, so this survey agreed with the previous data.

The plant genera with the greatest richness of galls were Eugenia L. (Myrtaceae) $(\mathrm{n}=5)$, Byrsonima Rich. ex Kunth (Malpighiaceae) $(\mathrm{n}=3)$, and Erythroxylum P. Browne (Erythroxylaceae) $(n=3)$. Similar results were found in other restinga areas of the State of Rio de Janeiro, where Eugenia and Erythroxylum (Erythroxylaceae) were pointed out as super host plants (Maia, 2001; Oliveira and Maia, 2005). Although, Byrsonima is cited in this condition for the first time.

Most galls were observed on leaves (77\%), a world pattern noted by Mani (1964), and confirmed by Maia (2013) for restinga areas and Gonçalves-Alvim and Fernandes (2001) for cerrado vegetation. Maia (2001) argues that the leaves are the most frequently attacked vegetal organ in restingas as they represent a constant and abundant source. The second most attacked plant organ was the stem. Similar results were presented by Gonçalves-Alvim and Fernandes (2001).

The plant organ specifity is confirmed, as suggested by Carneiro et al. (2009), as $92 \%$ of the gall morphotypes were recorded on a single organ.

The galls were classified into twelve different shapes, being the discoid, globoid and conical the most frequent. One-chambered galls (87\%) predominated, as well as glabrous galls (97). In other inventories, globoid, one-chambered, and glabrous galls were also indicated as one of the most common (Maia and Souza, 2013; Malves and Freiro-Costa, 2012; Saito and Urso-Guimarães, 2012; Bregonci et al., 2010, Santos et al., 2012), suggesting that these are the predominant morphologies of Brazilian galls.

Comparing the richness of each plant species among different restingas of the Southeastern region, we can realize that six botanical species showed the same number of gall morphotypes, 17 presented a smaller number, and three showed new records of gall morphotypes: Norantea brasiliensis Choisy (Marcgraviaceae), Clitoria ternatea L. and Clitoria laurifolia Poir (Fabaceae) (Table 6).

All records presented here are new, as this is the first survey of insect galls in the Restinga of Marambaia (RJ). Furthermore, Norantea brasiliensis Choisy (Marcgraviaceae), Clitoria ternatea L. and Clitoria laurifolia Poir (Fabaceae) are recorded as host plant of insect galls for the first time in restinga inventories. 
Table 6. Distribution of the total number of morphotypes of insect galls in restinga areas of the Southeastern region and number of morphotypes recorded from the Restinga de Marambaia), Rio de Janeiro, RJ.

\begin{tabular}{|c|c|c|}
\hline Host-plant & $\begin{array}{c}\text { Total } \\
\text { number of } \\
\text { morphotypes } \\
\text { of insects } \\
\text { galls } \\
\end{array}$ & $\begin{array}{c}\text { Number of } \\
\text { morphotypes } \\
\text { reported to } \\
\text { Restinga de } \\
\text { Marambaia } \\
\end{array}$ \\
\hline $\begin{array}{l}\text { Anacardium } \\
\text { occidentale }\end{array}$ & 01 & 01 \\
\hline $\begin{array}{l}\text { Aspidosperma } \\
\text { pyricollum }\end{array}$ & 01 & 01 \\
\hline Byrsonima sericea & 04 & 03 \\
\hline Clitoria laurifolia & 0 & 01 \\
\hline Clitoria ternatea & 0 & 01 \\
\hline $\begin{array}{l}\text { Dalbergia } \\
\text { ecastophylla }\end{array}$ & 01 & 01 \\
\hline $\begin{array}{l}\text { Erythroxylum } \\
\text { ovalifolium }\end{array}$ & 05 & 03 \\
\hline Eugenia astringens & 07 & 03 \\
\hline Eugenia uniflora & 06 & 02 \\
\hline Heteropteris nitida & 03 & 01 \\
\hline Fridericia conjugata & 03 & 02 \\
\hline Guapira opposita & 10 & 02 \\
\hline Inga maritima & 01 & 01 \\
\hline $\begin{array}{l}\text { Jacquemontia } \\
\text { holosericea }\end{array}$ & 01 & 01 \\
\hline Manilkara subsericea & 07 & 01 \\
\hline Maytenus obtusifolia & 02 & 01 \\
\hline $\begin{array}{l}\text { Neomitranthes } \\
\text { obscura }\end{array}$ & 06 & 01 \\
\hline Norantea brasiliensis & 0 & 01 \\
\hline Ouratea cuspidata & 03 & 01 \\
\hline Pouteria venosa & 01 & 01 \\
\hline $\begin{array}{l}\text { Schinnus } \\
\text { terebinthifolius }\end{array}$ & 02 & 01 \\
\hline Smilax rufescens & 06 & 01 \\
\hline
\end{tabular}

Table 7. Distribution of the number of morphotypes of insect galls in restinga areas of the Southeastern region of Brazil. Restinga de Marambaia, Barra de Guaratiba, Rio de Janeiro (RJ).

\begin{tabular}{lc}
\hline \multicolumn{1}{c}{ Localities } & $\begin{array}{c}\text { Number of gall } \\
\text { morphotypes }\end{array}$ \\
\hline Bertioga, SP & 233 \\
Maricá, RJ & 72 \\
Carapebus, RJ & 62 \\
Grumari, RJ & 43 \\
Arraial do Cabo, RJ & 41 \\
PEPCV, ES & 38 \\
RBEPS, RJ & 36 \\
Restinga de Marambaia, RJ & 31 \\
\hline
\end{tabular}

\section{Conclusion}

The richness of insect galls in the Restinga de Marambaia was lower when compared to other restinga areas of the southeastern Brazil (Table 7). However, the majority of the host plant species showed similar number of gall morphotypes (Table 6). The Fabaceae and Myrtaceae are the plant families with the greatest number of galls, and Erythroxylum ovalifolium Peyr. (Erytrhoxylaceae) and Eugenia astringens Cambess. (Myrtaceae) are the super-host plant species.

The majority of the gall morphotypes are glabrous, one-chambered, occur on leaves, and present organ specificity.

The guild of galling insects in the Restinga de Marambaia includes only three orders: Diptera, Hemiptera, and Lepidoptera, being less diverse than in most other areas of restinga. The majority of the galls are induced by Cecidomyiidae (Diptera), as in any other place in the world.

All gall records are new for the Restinga de Marambaia, Rio de Janeiro (RJ) and Norantea brasiliensis, Clitoria ternatea, and Clitoria laurifolia are for the first time recorded as host plant species in restinga inventories.

\section{Acknowledgements}

We are grateful to Coronel Franco, Head of the Social Communication from CAEx (Army Center Reviews) by support during the field work, to Museu Nacional-UFRJ for logistical support, and to $\mathrm{CNPq}$ for financial support.

\section{References}

AFONSO, A.S., MEDEIROS, A.S., NUNES, C.S., RODRIGUES, G.A., NUNES, R.S., TAVARES, L.F.M. and CONDE, M.M.S., 2007. Florística da vegetação arbustiva aberta na Restinga de Marambaia, RJ. Revista Brasileira de Biociencias, vol. 5, suppl. 2, pp. 450-452.

BREGONCI, J.M., POLYCARPO, P.V. and MAIA, V.C., 2010. Galhas de insetos do Parque Estadual Paulo César Vinha (Guarapari, ES, Brasil). Biota Neotropica, vol. 10, no. 1, pp. 265-274. http:// dx.doi.org/10.1590/S1676-06032010000100023.

CARNEIRO, M.A.A., BORGES, R.A.X., ARAÚJO, A.P.A. and FERNANDES, G.W., 2009. Insetos indutores de galhas da porção sul da Cadeia do Espinhaço, Minas Gerais, Brasil. Revista Brasileira de Entomologia, vol. 53, no. 4, pp. 570-592. http:// dx.doi.org/10.1590/S0085-56262009000400007.

COMANDO-GERAL DO CORPO DE FUSILEIROS NAVAIS - CGCFN, 2015 [viewed 7 May 2015]. Marambaia [online]. Rio de Janeiro: CGCFN. Available from: www.mar.mil.br/ cgcfn/marambaia

FERNANDES, S.P.C. and MAIA, V.C., 2011. Registros de galhas de insetos no município de Paraty (RJ, Brasil). São Paulo: Sociedade de Ecologia do Brasil.

GAGNÉ, R.J., 1994. The gall midges of the Neotropical Region. Ithaca: Comstock Cornell University Press. 352 p.

GONÇALVES-ALVIM, S.J. and FERNANDES, G.W., 2001. Comunidades de insetos galhadores (Insecta) em diferentes fisionomias do cerrado em Minas Gerais, Brasil. Revista 
Brasileira de Zoologia, vol. 18, suppl. 1, pp. 289-305. http:// dx.doi.org/10.1590/S0101-81752001000500025.

ISAIAS, R.M.S., CARNEIRO, R.G.S., OLIVEIRA, D.C. and SANTOS, J.C., 2013. Illustrated and annotated checklist of brazilian gall morphotypes. Neotropical Entomology, vol. 42, no. 3, pp. 230-239. http://dx.doi.org/10.1007/s13744-013-01157. PMid:23949804.

MAIA, V.C. and OLIVEIRA, J.C., 2010. Galhas de insetos da Reserva Biológica Estadual da Praia do Sul (Ilha Grande, Angra dos Reis, RJ). Biota Neotropica, vol. 10, no. 4, pp. 227-237. http:// dx.doi.org/10.1590/S1676-06032010000400028.

MAIA, V.C. and SOUZA, M.C., 2013. Insect galls of the xeric vegetation of Ilha do Cabo Frio (Arraial do Cabo, RJ, Brazil). Biota Neotropica, vol. 13, no. 3, pp. 278-288. http://dx.doi. org/10.1590/S1676-06032013000300030.

MAIA, V.C., 2001. The gall midges (Diptera, Cecidomyiidae) from three restingas of Rio de Janeiro State, Brazil. Revista Brasileira de Zoologia, vol. 18, no. 2, pp. 583-629. http://dx.doi. org/10.1590/S0101-81752001000200028.

MAIA, V.C., 2013. Galhas de insetos em restingas da região sudeste do Brasil com novos registros. Biota Neotropica, vol. 13, no. 1, pp. 183-209. http://dx.doi.org/10.1590/S1676-06032013000100021.

MAIA, V.C., MAGENTA, M.A.G. and MARTINS, S.E., 2008. Ocorrência e caracterização de galhas de insetos em áreas de restinga de Bertioga (São Paulo, Brasil). Biota Neotropica, vol. 8, no. 1, pp. 167-197. http://dx.doi.org/10.1590/S1676-06032008000100020.

MALVES, K. and FREIRO-COSTA, A. F., 2012. List of Plants with Galls Induced by Insects from the UNILAVRAS/Boqueirão Biological Reserve, Ingaí, state of Minas Gerais, Brazil. Check List, vol. 8, no. 3, pp. 426-431.

MANI, M.S., 1964. Ecology of plant galls. The Hague: Junk. 434 p. http://dx.doi.org/10.1007/978-94-017-6230-4.

MONTEIRO, R.F., FERRAZ, F.F.F., MAIA, V.C. and AZEVEDO, M.A.P., 1994. Galhas entomógenas em restingas: uma abordagem preliminar. In: S. WATANABE, ed. Anais do III Simpósio de Ecossistemas da Costa Brasileira: subsídios a um gerenciamento ambiental. São Paulo: ACIESP, pp. 210-220. vol. 3.
MONTEIRO, R.F., ODA, R.A.M., NARAHARA, K.L. and CONSTANTINO, A.L., 2004. Galhas: diversidade, especificidade e distribuição. In: C.F.D. ROCHA, F.A. ESTEVES and F.R. SCARANO. Pesquisa de longa duração na Restinga de Jurubatiba: ecologia, história natural e conservação. Macaé: NUPEM/ UFRJ, pp. 127-141.

OLIVEIRA, J.C. and MAIA, V.C., 2005. Ocorrência e Caracterização de Galhas de Insetos na Restinga de Grumari (Rio de Janeiro, RJ, Brasil). Arquivos do Museu Nacional, vol. 63, no. 4, pp. 669-675.

RIO DE JANEIRO, 2013. Decreto 37483, de 31 de julho de 2013, de Área de Especial Interesse Ambiental (AEIA) da Região de Guaratiba - XXVI RA. Diário Oficial do Município do Rio de Janeiro, Rio de Janeiro, 01 aug.

RODRIGUES, A.R. and MAIA, V.C., 2010. Duas novas espécies de Lopesia Rübsaamen (Diptera, Cecidomyiidae) do Brasil, com chave para as espécies. Biota Neotropica, vol. 10, no. 1, pp. 85-99. http://dx.doi.org/10.1590/S1676-06032010000100008.

RODRIGUES, A.R. and SILVA, S.A.H., 2011 [viewed 16 April 2015]. Galhas entomógenas associadas à Myrtaceae da Ilha da Marambaia (Mangaratiba, RJ) [online]. São Paulo: Sociedade de Ecologia do Brasil. Available from: www.sebecologia.org.br/ xceb/resumos/447.pdf

SAITO, V.S. and URSO-GUIMARÃES, M.V., 2012. Characterization of galls, insect galls and associated fauna of Ecological Station of Jataí (Luiz Antônio, SP). Biota Neotropica, vol. 12, no. 3, pp. 99-107. http://dx.doi.org/10.1590/S1676-06032012000300011.

SANTOS, J.C., ALMEIDA-CORTEZ, J.S. and FERNANDES, G.W., 2012. Richness of gall-inducing insects in the tropical dry forest (caatinga) of Pernambuco. Revista Brasileira de Entomologia, vol. 55, no. 1, pp. 45-54. http://dx.doi.org/10.1590/ S0085-56262011000100009.

SHORTHOUSE, J.D., WOOL, D. and RAMAN, A., 2005. Gallinducing insects: nature's most sophisticated herbivores. Basic and Applied Ecology, vol. 6, no. 5, pp. 407-411. http://dx.doi. org/10.1016/j.baae.2005.07.001.

STONE, G.N. and SCHÖNROGGE, K., 2003. The adaptive significance of insect gall morphology. Trends in Ecology \& Evolution, vol. 18, no. 10, pp. 512-522. http://dx.doi.org/10.1016/ S0169-5347(03)00247-7. 